\title{
Importancia económica de las ciudades medias en México
}

\section{Mexican Medium-Sized Cities' Economic Importance}

\author{
Mario Alberto García Meza* (D) https://orcid.org/0000-0001-7292-7110 \\ Ana Lilia Valderrama Santibáñez * (D) https://orcid.org/0000-0003-0372-7099 \\ Omar Neme Castillo** (i) https://orcid.org/0000-0001-8509-7937
}

\section{Resumen}

El crecimiento urbano en México ha conducido a una participación económica más equitativa de las ciudades de acuerdo con el tamaño de su población. En particular, las ciudades de tamaño medio de población han ganado peso económico. El objetivo del presente trabajo es analizar la importancia de este tipo de aglomeraciones urbanas en la economía del país durante 2000 y 2010 . Esto se prueba a través de la metodología planteada por la ley de Zipf, que mide la distribución del tamaño - económico- de las ciudades por el coeficiente de Pareto. En términos econométricos, se emplean mínimos cuadrados ordinarios (MCO) con errores estándar robustos y se incluye toda la gama de ciudades considerando regresiones de rangos móviles. Los resultados permiten aceptar la hipótesis de un coeficiente de Pareto menor a la unidad en ambos años para submuestras de mayor tamaño, rechazando, por tanto, la ley de Zipf. En este sentido, se argumenta que las ciudades de tamaño medio de población tienen mayor peso económico relativo que el de cualquier otro tipo de aglomeración urbana.

Palabras clave: ciudades medias; distribución de ciudades; PIB por ciudades; ley de Zipf.

\begin{abstract}
Mexican urban growth has led to a more equative participation of cities accordingly to their population, where medium-sized cities particularly have gain economic weight. The aim or the paper is to analyse the relevance of this kind of urban agglomerations in the country's economy in 2000 and 20I0. The methodology set up by Zipf's law is used, which measures city size distribution - as measured by economic indicators - through Pareto's coefficient. Econometrically, ordinary least squares (OLS) with robust standard errors are followed, and all cities' range is included using moving average regressions. The results accept the hypothesis of a Pareto's coefficient below I in both years for equal size subsamples. In consequence, Zipf's law is rejected. In this sense, the relative higher economic weight of medium-sized cities is accepted.

Keywords: Medium-sized cities; city distribution; gross domestic product (GDP) by cities; Zipf's law.
\end{abstract}

Cómo citar: García Meza, M.A.,Valderrama Santibáñez,A. L., y Neme Castillo, O. (2019). Importancia económica de las ciudades medias en México. región y sociedad, 3I, el 24I. doi: I0.22198/rys20I9/3I/I24I

*Autor para correspondencia. Universidad Juárez del Estado de Durango, Facultad de Economía, Contaduría y Administración. Fanny Anitúa y Privada de la Loza s. n., C. P. 34000. Durango, Durango, México. Correo electrónico: mario.agm@ujed.mx

** Instituto Politécnico Nacional, Escuela Superior de Economía, Sección de Estudios de Posgrado e Investigación. Plan de Agua Prieta Núm. 66, Col. Plutarco Elías Calles, Unidad Profesional Adolfo López Mateos,

Casco de Santo Tomás, Alcaldía Miguel Hidalgo, C. P. 11340, Ciudad de México. Correo electrónico: avalderrama@ipn.mx

*** Instituto Politécnico Nacional, Escuela Superior de Economía, Sección de Estudios de Posgrado e Investigación. Plan de Agua Prieta Núm. 66, Col. Plutarco Elías Calles, Unidad Profesional Adolfo López Mateos,

Casco de Santo Tomás, Alcaldía Miguel Hidalgo, C. P. 11340, Ciudad de México. Correo electrónico: oneme@ipn.mx

Recibido: 28 de agosto de 2019

Aceptado: 19 de noviembre de 2019

Liberado: 18 de diciembre de 2019

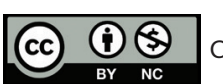

Esta obra está protegida bajo una Licencia

Creative Commons Atribución-No Comercial 4.0 Internacional. 


\section{Introducción}

Las ciudades son el modo de organización social más duradero y estable de la humanidad. Conglomeran los grupos demográficos y económicos dominantes del mundo (Khanna, 2016) y concentran actividades industriales, de generación del conocimiento, de inversión y de innovación, entre otras.

Al respecto, el éxito de las ciudades grandes está documentado en la literatura. Sin embargo, las ciudades medias, aquellas en un rango de población de entre 500 mil y 1 millón de habitantes, emergen con mayor potencial para liderar un futuro económico inclusivo y sustentable que reduzca la brecha entre lo urbano y lo rural (McFarland, 2017). A pesar de la visibilidad de las grandes ciudades, la mayoría de la población y del crecimiento urbano se presenta en ciudades medias y pequeñas (Banco Mundial [BM], 2015).

Para Clark y Clark (2014), en los sistemas de ciudades se generan desplazamientos de la población, de recursos y de bienes y se despliegan servicios, por lo que existen complementariedades y competencias entre ciudades, las cuales son la base del sistema económico. En estos sistemas, los grandes centros poblacionales funcionan a escala nacional e internacional, mientras que las ciudades medias ocupan un lugar vinculante entre las grandes y pequeñas aglomeraciones - urbanas y rurales-.

La importancia de las ciudades medias en la economía mexicana puede juzgarse si se considera su papel dentro de la red de ciudades. En 2000 y 2010, el crecimiento de la población y del producto interno bruto (PIB) en estas ciudades fue, en términos relativos, mayor que el de cualquier otro tipo de aglomeración urbana. Este grupo de ciudades aglutinó, en 2010, 16.3 millones de personas, equivalentes a $18 \%$ de la población urbana, y representó $23.3 \%$ del $\mathrm{PIB}$, en ambos casos similar al de las grandes ciudades, lo cual sugiere un peso económico relativamente proporcional entre ciudades grandes y medias. De este modo, se vislumbra una distribución por tamaño de ciudad más equitativa; en otras palabras, que la participación económica de las ciudades medias, como segunda categoría de relevancia en el sistema urbano nacional, es proporcionalmente mayor.

Existe una tradición empírica urbana (Parr y Jones, 1983; Rosen y Resnick, 1980) que describe el tamaño de la distribución de las ciudades en el interior de los sistemas urbanos y su importancia en la jerarquía urbana. En particular, Auerbach (1913, citado en Giesen y Suedekum, 2009) argumenta que el tamaño de las ciudades tiene una forma similar a una distribución de Pareto, en la que un reducido número de ciudades concentra una elevada proporción de población o "masa", y un gran número de ciudades agrupan una reducida proporción de ésta.

En este sentido, el objetivo del presente artículo es analizar la importancia de las ciudades medias en la vida económica del país. Así, el aparente mayor peso de las ciudades medias se prueba mediante una distribución particular de Pareto conocida como ley de Zipf. Se analizan, teniendo en cuenta los años 2000 y 2010 dada la disponibilidad de datos, 25 ciudades que pueden considerarse como medias. Se establece la hipótesis según la cual el comportamiento 
de las ciudades medias representa un mecanismo subyacente que impulsa la actividad económica (coeficiente de Pareto menor a la unidad).

El documento se estructura en cinco secciones. En las siguientes se revisa la literatura empírica sobre el tema. Después se caracterizan las ciudades medias, se describe la metodología y se estima la distribución del tamaño económico de las ciudades. Al final se presentan las conclusiones.

\section{Economía de las ciudades medias}

Los supuestos políticos, económicos y sociales respecto a la capacidad de las ciudades para impulsar la actividad económica se originan en la literatura académica, sobre todo europea y estadunidense. Entre los aspectos clave sobresalen: 1) las ciudades albergan a más de la mitad de la población mundial y son los “motores de la innovación" (Glaeser, 2011); 2) la menor importancia relativa de la distancia, derivada de los avances tecnológicos, permite que las ciudades sean lugares óptimos para trabajar y disfrutar de la vida (Glaeser, 2011); 3) existen procesos de descentralización y desconcentración política que llevan el poder hacia las principales ciudades (Katz y Bradley, 2013).

Estos principios se basan en paradigmas económicos, sociales y urbanos asociados a ideas tanto de la Escuela de Chicago, como a la nueva geografía económica (NGE) combinada con elementos de la economía urbana (véase, por ejemplo, Florida, 2002; Henderson y Thisse, 2004; Krugman, 1991). La NGE trata de explicar la estructura espacial de la economía y, en particular, la concentración espacial de la actividad económica en las ciudades. Concibe el fenómeno de aglomeración como resultado de un proceso de causalidad circular, impulsado por externalidades pecuniarias dadas las economías de escala en la producción y distribución. Los conceptos centrales se resumen en: 1) maximización individual: empresas y trabajadores determinan dónde ubicarse de acuerdo con los costos de transacción relativos (trasporte, acceso a bienes, servicios y mano de obra) y con la movilidad de sus mercados. Cuando los mercados pueden atenderse a bajo costo a la distancia, tienden a aglomerarse formándose ciudades; 2) fuerza magnética: debido a la competencia imperfecta, las fuerzas de aglomeración prevalecen sobre las de dispersión espacial, de modo que se concentran las personas y el capital en centros urbanos, alimentando la aglomeración, ya que empresas y trabajadores buscan ubicarse donde la demanda laboral y los ingresos sean más altos; y 3 ) equilibrio: la aglomeración aumenta los efectos spillovers (desbordamiento) localizados, por ejemplo, investigación, desarrollo e innovación que incentivan la productividad promedio de ese espacio. Con el tiempo, los efectos positivos se mueven a un estado estable, ya que las nuevas entradas al mercado aumentan la competencia, y los efectos de congestión generan externalidades negativas.

Está claro que las ciudades están creciendo poblacional, territorial y físicamente. Hechos como que las empresas tiendan a agruparse, de manera natural o planificada, que los trabajadores con habilidades similares se concentren en lugares particulares, o que los ingresos más altos se paguen en centros urbanos, 
son incentivos que tienden a compensar las "externalidades negativas" que se sufren al habitar o trabajar en ciertas ciudades que, por definición, son centros de fricciones.

En términos de evidencia empírica, los estudios han demostrado que en países industrializados, el tamaño de las ciudades es mucho menos importante que su conectividad global (McCann y Acs, 2011); que duplicar su tamaño genera un aumento de entre 4 y $7 \%$ del PIB per cápita (Rosenthal y Strange, 2004); que los trabajadores bien calificados y los creativos tienden a moverse hacia y entre las grandes ciudades (Florida, 2002); que dominan en los índices de generación de conocimiento (McCann y Acs, 2011), base de la productividad, de la competencia y del crecimiento; que las mayores tienden a concentrar la población y la riqueza (Core Cities, 2013), generan más de 70\% del PIB mundial, lo cual propicia la migración dado el diferencial entre salarios urbanos y rurales (ONU-Habitat, 2010); que el crecimiento nacional se beneficia de una apropiada distribución espacial de la actividad económica, por lo general centrada en las grandes (Bajpai y Muzzini, 2016); que las de mayor tamaño, a pesar de su dinamismo económico, pueden no funcionar como motores de crecimiento en ausencia de las secundarias prósperas (Duranton, 2015); que las fronterizas y las que están en los corredores económicos particularmente dinámicos se benefician de las inversiones en la industria, al tiempo que las medias, que están creciendo más rápido que el promedio nacional, se localizan en las afueras de las aglomeraciones más grandes (Cadena, Remes y Manyika, 2011); que en términos geográficos, el ciclo de producción se ha fragmentado a escala global localizándose principalmente en centros urbanos con ventajas en costos (Guerrero, Lucenti y Galarza, 2009); que las de tamaño medio muestran creciente competitividad en comparación con las más grandes (Henderson, 2000); y que las pequeñas y medias tienden a ser más productivas que las grandes (Organización para la Cooperación y Desarrollo Económicos [OCDE], 2006).

En particular, existe evidencia de la relevancia de las ciudades medias sobre distintas dimensiones económicas. Por ejemplo, según McCann y Acs (2011) y Dijkstra, Garcilazo y McCann (2013), los centros urbanos medios y pequeños contribuyen de manera significativa a la economía nacional. De acuerdo con Bolay y Ravinovich (2004), este tipo de ciudades tienen actividades locales y regionales notables que las posicionan como actores de los cambios social y económico de los países en desarrollo. Al respecto, Zul, Hudalah, Rahayu y Woltjer (2014) señalan que la configuración de las ciudades medias permite que los agentes urbanos y económicos (trabajadores, migrantes, empleadores, funcionarios públicos y servicios públicos) se relacionen de manera eficiente para impulsar el empleo y la rentabilidad frente a los grandes centros urbanos.

Si bien esta idea puede aplicarse a aglomeraciones de diferente tamaño, Weziak (2016) argumenta que la satisfacción con la vida en una ciudad aumenta con el tamaño de la comunidad. Sin embargo, la insatisfacción crece con las ineficiencias relacionadas con el trasporte público, con el acceso a espacios culturales y deportivos, con la disponibilidad de puntos de venta, de espacios verdes, de oficinas públicas, con la calidad del aire y la confianza en las personas, que en muchos casos se presenta por efectos de la congestión poblacional. Da- 
das las externalidades negativas (desigualdades, inseguridad, contaminación, fragmentación) que emergen con el tamaño, Duroudier (2014) considera que el tipo de ciudad ideal, con mejores condiciones sociales, económicas, políticas y ambientales, tienden a ser el centro urbano de tamaño medio.

En éstas, la menor escala representa oportunidades para que los extremos sociales y económicos coexistan y se establezcan sinergias, mientras que, en las grandes ciudades, la distancia es tal-por los mercados inmobiliarios y las estrategias de autosegregación y de discriminación de mercado- que los efectos negativos tienden a perpetuarse. En concreto, Klaufus (2010) afirma que las comunidades heterogéneas y los espacios públicos multifuncionales accesibles son cualidades positivas de las ciudades pequeñas y medias, que representan una potencialidad para el desarrollo urbano sostenible (Klaufus, 2010).

Así mismo, Camagni, Capello y Caragliu (2013) identifican dos tipos de factores específicos que permiten a las ciudades medias competir con las grandes. Primero, los efectos negativos de la aglomeración se incrementan con el tamaño, por lo que tienden a gestionarse mejor en ciudades medias. Segundo, el tamaño por sí solo no justifica la posición competitiva ni la función de un centro urbano. Así, la organización espacial de las ciudades influye en su eficiencia, crecimiento, productividad y especialización. El menor tamaño, la proximidad y la conectividad con la gran ciudad, con factores institucionales y con el patrimonio económico, sustentan la competitividad de las ciudades medias (Puissant y Lacour, 2011).

Además, mediante el empleo de recursos urbanos y territoriales, las ciudades medias tienden a ser las aglomeraciones urbanas más productivas, por arriba de las grandes ciudades (McCann y Acs, 2011). Al respecto, entre los países ricos, doce de las quince ciudades más emprendedoras son pequeñas y medianas (Acs, Desai y Hessels, 2008), y once de las quince ciudades más competitivas del mundo también son pequeñas y medianas (ATKearney, 2017). En este sentido, Fritsch y Mueller (2004) sugieren que el espíritu empresarial mejora de manera significativa el desarrollo económico en ciudades medianas y grandes, aunque los efectos indirectos positivos sólo se generan en las últimas debido al requisito del umbral mínimo de mercado (Audretsch, Belitski, y Desai, 2015). No obstante, Grabher (2018) señala que las actividades innovadoras y emprendedoras también se presentan en ciudades pequeñas y medias.

Respecto de las inversiones, Sridhar y Wan (2010) observan que las ciudades capitales o de mayor tamaño tienden a ser menos atractivas para que se asienten las empresas. En particular, en las economías en desarrollo, las empresas intensivas en mano de obra tienden a ubicarse en ciudades medias o grandes, no en mega ciudades, porque el aumento de salarios y los costos de capacitación se encuentran en niveles promedio. Así, las empresas favorecen la sostenibilidad del empleo basándose en las ventajas únicas de las ciudades medias. Aún más, la ubicación de las empresas nacionales envía señales a las empresas multinacionales que muestran el área como un buen lugar para los negocios y la calidad de vida de sus empleados, lo cual refuerza el potencial de las ciudades medias. 
El Programa de Naciones Unidas para los Asentamientos Humanos (ONU-Habitat, 2010) afirma que, en general, las ciudades pequeñas y medianas desempeñan una variedad de funciones: 1) sirven como centros de crecimiento económico locales, es decir, mercados para productos rurales y servicios urbanos; 2) actúan como puentes entre las áreas rurales y los grandes centros urbanos; 3) algunas funcionan como sede de la administración municipal; y 4) sirven como escalones para los migrantes rurales en su camino hacia otros destinos. ${ }^{1}$ Por lo tanto, como señalan Berdegué y Soloaga (2018), el crecimiento de estas ciudades impulsa el desarrollo rural y urbano de otros centros poblacionales.

En este sentido, la posición "puente" de una ciudad dentro del sistema urbano deriva de una compleja interacción de condiciones económicas, geográficas y socioculturales, determinadas parcialmente a escala local. Cox y Longlands (2016) identifican una serie de factores que permiten comprender el funcionamiento de un sistema urbano y el rol de las ciudades medias. Lugares diferentes desempeñan roles diferentes y complementarios dentro de una jerarquía urbana. Las ciudades se relacionan entre sí en términos económicos y poblacionales; por ejemplo, los patrones de trasporte y la conectividad facilitan la movilidad de personas, de bienes e insumos, por lo que los mercados laborales, de bienes y de servicios están interconectados mediante una cadena de valor-suministro. No obstante, siempre existe un lugar central que tiende a concentrar los factores y los procesos productivos, y a determinar la dinámica económica regional. Jones, Bhatta, Gill, Pantuliano, Singh, Timsina, Uppadhaya y Walker (2009) encuentran que el fortalecimiento de los vínculos económicos entre los centros urbanos puede contribuir al crecimiento económico sostenible, a una mayor prosperidad individual, a la atracción y retención de trabajadores más calificados y a la reducción de las privaciones. Dicho fortalecimiento es entonces un mecanismo de desarrollo económico local.

De este modo, la creación de vínculos entre los centros económicos -que contienen mayores tasas de empleo y empresas más grandes, dinámicas y diversificadas - y las ciudades circundantes de menor tamaño, estimula el crecimiento económico. En este sentido, tienden a generarse efectos spillovers desde las primeras hacia las segundas, hasta que se alcanzan los efectos de aglomeración en los lugares centrales (Jones et al., 2009).

Así mismo, muchos trabajos ubicados en estas ciudades son respaldados de manera directa por la demanda de empresas y consumidores en las áreas circundantes, entre las que se incluyen ciudades de menor tamaño. Entre las actividades y empleos vinculados están el comercio minorista, las actividades recreativas, culturales, los servicios empresariales, de educación y de salud. A este respecto, la escala y la prosperidad del hinterland (área de influencia) actúan como motores del crecimiento de la ciudad. En otras palabras, la ciudad central sigue la dinámica que sucede en otros lugares, y no sólo el crecimiento interno.

1 A pesar de que las ciudades pequeñas y medianas tienen una infraestructura deficiente, como abastecimiento de agua y saneamiento inadecuados, recolección de desechos sólidos irregulares, caminos sin pavimentar, suministro eléctrico errático y mala conectividad de teléfono e internet, tienen un importante papel vinculante entre las economías rurales y las economías urbanas (Dahiya, 2012). 
En concreto, las ciudades no pueden ser tratadas como entes aislados, y sus interrelaciones con otros lugares representan ventajas mutuas que deben explotarse desde la política pública (Cheshire, Magrini, Medda y Monastiriotis, 2004). Así, las ciudades y sus zonas de influencia tienen una relación de interdependencia con respecto a la ubicación, la integración espacial, las estructuras sociales y económicas, las relaciones con otras ciudades, y a las características particulares del proceso de urbanización en cada una de ellas (Bolay y Ravinovich, 2004). Desde esta óptica, la relevancia económica de las ciudades medias puede explicarse por el papel que desempeñan en las relaciones regionales, esto es, del establecimiento de vínculos con los lugares vecinos (Pike, Rodriguez y Tomaney, 2006).

Cuando las ciudades medias se conectan físicamente con otras aglomeraciones urbanas vecinas, sus funciones, actividades, territorios y roles se amplían. Al mantener altos niveles de integración social, económica, cultural, política y ambiental, se crean áreas o zonas metropolitanas que potencian sus efectos positivos. Así, las ciudades medias que operan dentro de este tipo de zonas adquieren una posición central en la jerarquía de ciudades, lo que se asocia también con su relevancia económica en el agregado regional y nacional.

Por otro lado, si bien destaca la importancia de las ciudades medias, también existen desafíos que deben enfrentar para avanzar en su desarrollo. El mayor de todos es que tienden a estar aisladas o las ciudades más grandes o dominantes las eclipsan, lo cual se explica por la dependencia económica histórica, por el capital humano limitado, la migración y el envejecimiento, la reputación local, la conectividad restringida y la debilidad institucional (Pike et al., 2016).

\section{Caracterización de las ciudades medias}

Desde la década de 1980, tras las reformas económicas implementadas, el país ha acelerado los procesos de urbanización, lo que ha ocasionado que la mayoría de la población habite en zonas urbanas. Este proceso de urbanización implica que las comunidades locales crezcan y se especialicen (Fang, Pang y Liu, 2017), aunque con niveles de eficiencia cuestionables.

Así, con la apertura del mercado doméstico, se registran crecimientos económicos importantes con tasas anuales promedio de $2 \%$ en el período 2000 2015. Al mismo tiempo, se da una rápida expansión urbana que modifica la composición geográfica de la población. En la década de 1980 , sólo $66.3 \%$ de la población vivía en zonas urbanas. En contraste, en 2015 la población urbana representó $79.2 \%$ del total nacional. Este valor es superior al promedio mundial (53.8\%) y ligeramente menor al promedio de los países desarrollados (81.2\%).

El perfil urbano e industrial al inicio de la década de 1980 condujo a una nueva etapa en el proceso de urbanización caracterizada por seis elementos: 1) disminución del peso relativo de la zona metropolitana de la Ciudad de México frente al resto del sistema urbano nacional; 2) descenso demográfico de las cuatro zonas metropolitanas más importantes (Ciudad de México, Guadalajara, Monterrey y Puebla; 3) movimientos demográficos intrametropolitanos tipo 
centro-periferia; 4) reorientación de flujos migratorios interurbanos e interregionales; 5) mayor crecimiento relativo en ciudades de menor tamaño; y 6) redistribución de la población sobre el territorio (Sobrino, 1999).

En este sentido, la rápida urbanización se aprecia en el incremento del número de ciudades. En la tabla 1 se muestra el porcentaje de población urbana y el número de ciudades de acuerdo con seis categorías del tamaño poblacional propuestas por la División de Población de la ONU, a saber: 1) de 10 millones o más; 2) de 5 a 10 millones; 3) de 1 a 5 millones; 4) de 500 mil a 1 millón; 5) de 100 mil a 500 mil; y 6) de 15 mil a 100 mil habitantes. Cabe señalar que este documento considera datos de ciudades individuales y no de zonas metropolitanas o concentraciones urbanas consolidadas en ciudades mayores. ${ }^{2}$

Al final del año 2000, se registraron 484 ciudades de al menos 15 mil habitantes, agrupando 60.04 millones de personas; en 2010 alcanzó 575 ciudades y concentra 68.68 millones de personas. La población en este tipo de zonas urbanas registró una tasa de crecimiento promedio anual de 1.35\%, para ubicarse entre las tasas de crecimiento poblacional total y total urbana.

Considerando el peso relativo de la población urbana por tamaño de ciudad, se tiene que las ciudades de mayor relevancia en 2000 fueron las clasificadas en el rango de 15 mil a 100 mil habitantes (23.15\%), seguidas por las ciudades de entre 1 y 5 millones de personas (22.04\%) y por aquéllas de entre 500 mil y 1 millón de habitantes (20.75\%). En 2010, se presenta un cambio en la distribución poblacional. Así, las de mayor relevancia son las ciudades con población en el rango de 500 mil a 1 millón (23.82\%), seguidas por ciudades con menos de 100 mil habitantes (22.21\%) y por aquéllas de entre 1 y 5 millones de habitantes (21.59\%).

Lo anterior indica que el país ha experimentado un notable crecimiento urbano, en particular en las ciudades medias, con crecimientos por arriba del promedio, ganando, en consecuencia, una importancia relativa en el sistema urbano nacional. Además, se observa una pérdida de importancia relativa de las grandes ciudades. Además, con los datos considerados hasta 2010, las ciudades que cumplen este criterio poblacional son 27 , con una población total de 10.6 millones dentro de sus límites.

Al mismo tiempo, la tabla 2 muestra el PIB agrupado por categorías de ciudades y su participación en el total. Dada la disponibilidad de datos, se calcula el PIB a escala municipal de esos años siguiendo la metodología propuesta por González y Gallegos (2014), basada en la población municipal activa en términos económicos y el PIB de cada entidad federativa. Las ciudades mayores contribuyen con $24.8 \%$ del PIB, mientras que las ciudades medias representan

2 Si bien este enfoque se aparta de la noción sistémica de Bourne (1982), donde las subáreas que conforman la ciudad constituyen una sola, Oliveras y Cartanyá (2015) señalan que la nueva realidad urbana se caracteriza por la intensificación de núcleos urbanos, homogéneos y heterogéneos, integrados y desintegrados socialmente, consecuencia de la descentralización y desterritorialización, como es el caso particular de Ciudad de México (Hiernaux y Lindón, 2004). Así, la estructura urbana interna de las ciudades medias pasa de una concéntrica a otra multicéntrica o sectorial, con nuevos patrones espaciales de funcionamiento (Álvarez, 2017) que favorecen que las subáreas urbanas menores, entendidas en este caso como alcaldías o municipios en zonas metropolitanas, tengan mayor presencia individual en el sistema de ciudades por sus conexiones desde la periferia. A través de esta perspectiva, las ciudades medias, consideradas de manera individual, muestran dinámicas socioeconómicas propias, si bien dentro de un contexto mayor, que descentralizan el sistema urbano. 


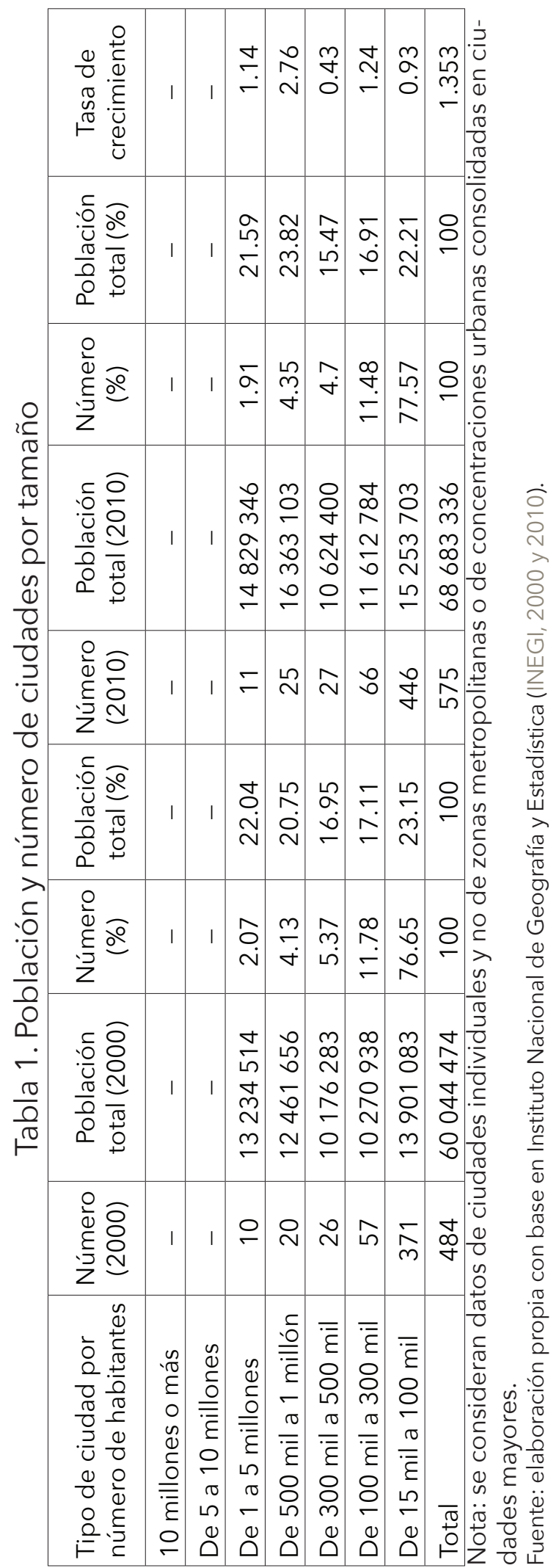


23.4\% del PIB en 2010. Las ciudades con menor participación son las de menor rango, aunque registran el mayor avance en la contribución al PIB urbano.

En concreto, el principal tipo de ciudad generadora de PIB es la grande, pero muy de cerca están las ciudades medias. En consecuencia, la importancia de las ciudades medias en el producto agregado es más que proporcional a la importancia de las ciudades mayores, lo cual sugiere una distorsión en la distribución del tamaño de ciudades que, en última instancia, condiciona el desempeño económico agregado y per cápita a escala nacional.

Dos aspectos a resaltar son, por un lado, que el peso económico del grupo de ciudades medias es similar al de las ciudades mayores (apenas 1.5 puntos porcentuales menor), y, por otro, que la tasa de crecimiento media anual de las primeras es mayor que el de las ciudades grandes durante el periodo de 2000 a 2010, lo que sugiere que este grupo está ganando relevancia en la vida socioeconómica del país. No obstante, las ciudades de entre 300 mil y 500 mil habitantes muestran el PIB per cápita promedio más alto en 2010 al pasar de 75 mil 607 pesos a 132 mil 695 pesos. Las grandes ciudades ocupan el segundo lugar considerando el PIB per cápita (131 mil 796 pesos), seguidas por las ciudades medias (112 mil 006 pesos).

Tabla 2. PIB por ciudades

\begin{tabular}{|l|c|c|c|c|}
\hline $\begin{array}{l}\text { Tipo de ciudad por } \\
\text { número de habitantes }\end{array}$ & PIB (2000) & Participación & PIB (2010) & Participación \\
\hline 10 millones o más & - & - & - & \\
\hline 5 a 10 millones & - & - & - & \\
\hline 1 a 5 millones & 974525.06 & 26.58 & 2039193.62 & 24.82 \\
\hline 500 mil a 1 millón & 908425.25 & 24.78 & 1918207.99 & 23.34 \\
\hline 300 mil a 500 mil & 704148.33 & 19.21 & 1531828.10 & 18.64 \\
\hline 100 mil a 300 mil & 551723.44 & 15.05 & 1403516.31 & 17.08 \\
\hline 15 mil a 100 mil & 527148.46 & 14.38 & 1324074.82 & 16.12 \\
\hline Total & 3665970.54 & 100.00 & 8216820.84 & 100.00 \\
\hline
\end{tabular}

Fuente: elaboración propia con base en el Sistema de Cuentas Nacionales, INEGI. Estimaciones del PIB municipal con base en González y Gallegos (2014).

Las ciudades medias se muestran en la tabla 3, ordenadas de mayor a menor PIB de los años 2000 y 2010 a precios de 2010. Como se señaló, existe un total de 25 ciudades intermedias distribuidas en 19 entidades federativas. La población de estas aglomeraciones oscila entre 809 mil (Chihuahua, Chihuahua) y 519 mil habitantes (Durango, Durango). La ciudad de mayor tamaño económico, es decir, con mayor PIB, es Álvaro Obregón, ${ }^{3}$ Ciudad de México (189 mil millones

3 En un sentido estricto, las ciudades que componen la Ciudad de México son parte de una misma mancha urbana. Éstas se solían dividir en delegaciones de un solo distrito federal. En la actualidad, éstas se han reclasificado como municipios de una misma entidad federativa, pero la interdependencia permanece. Con el objetivo de mantener consistencia con las definiciones establecidas en el presente documento sobre ciudades medias, estos municipios se mantienen en el análisis. 


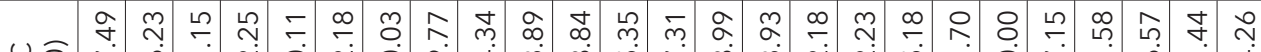

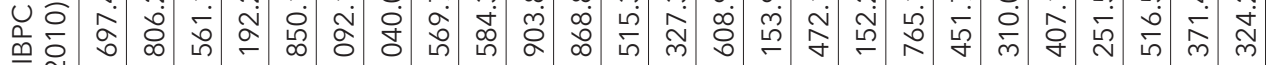

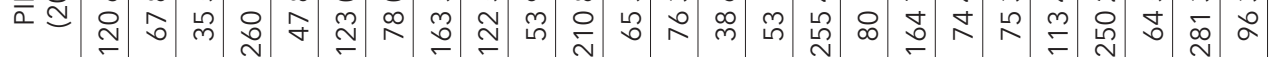
-

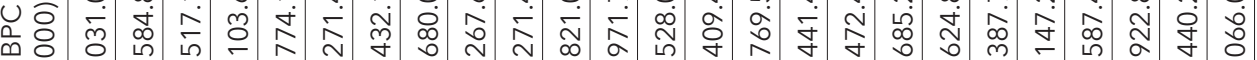

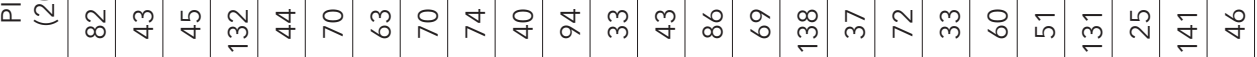

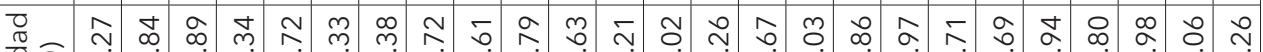

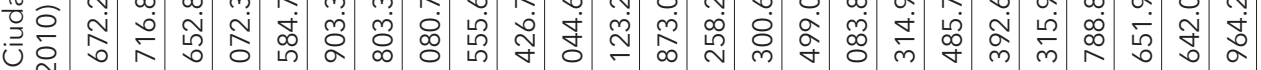

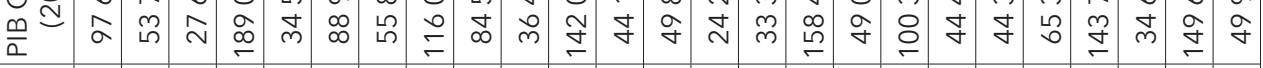

茜

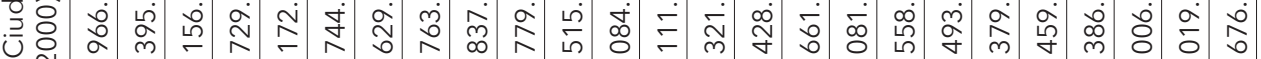

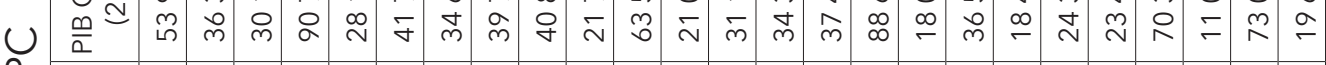

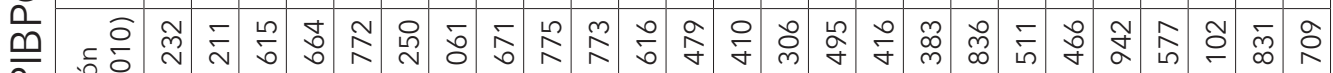

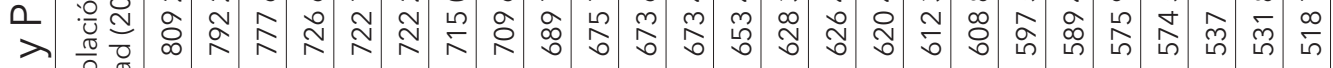

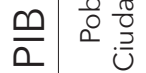

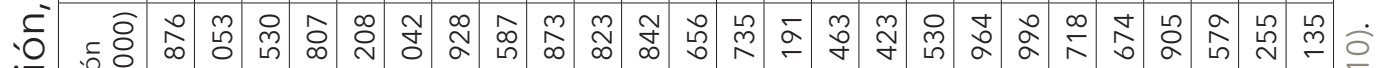

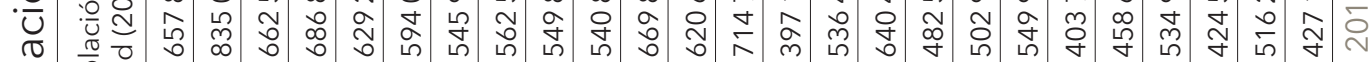

\begin{tabular}{c}
$\frac{0}{0}$ \\
\hline 0 \\
0 \\
0 \\
$\frac{0}{0}$ \\
0 \\
0 \\
0 \\
0 \\
0 \\
0 \\
0 \\
0 \\
0
\end{tabular}
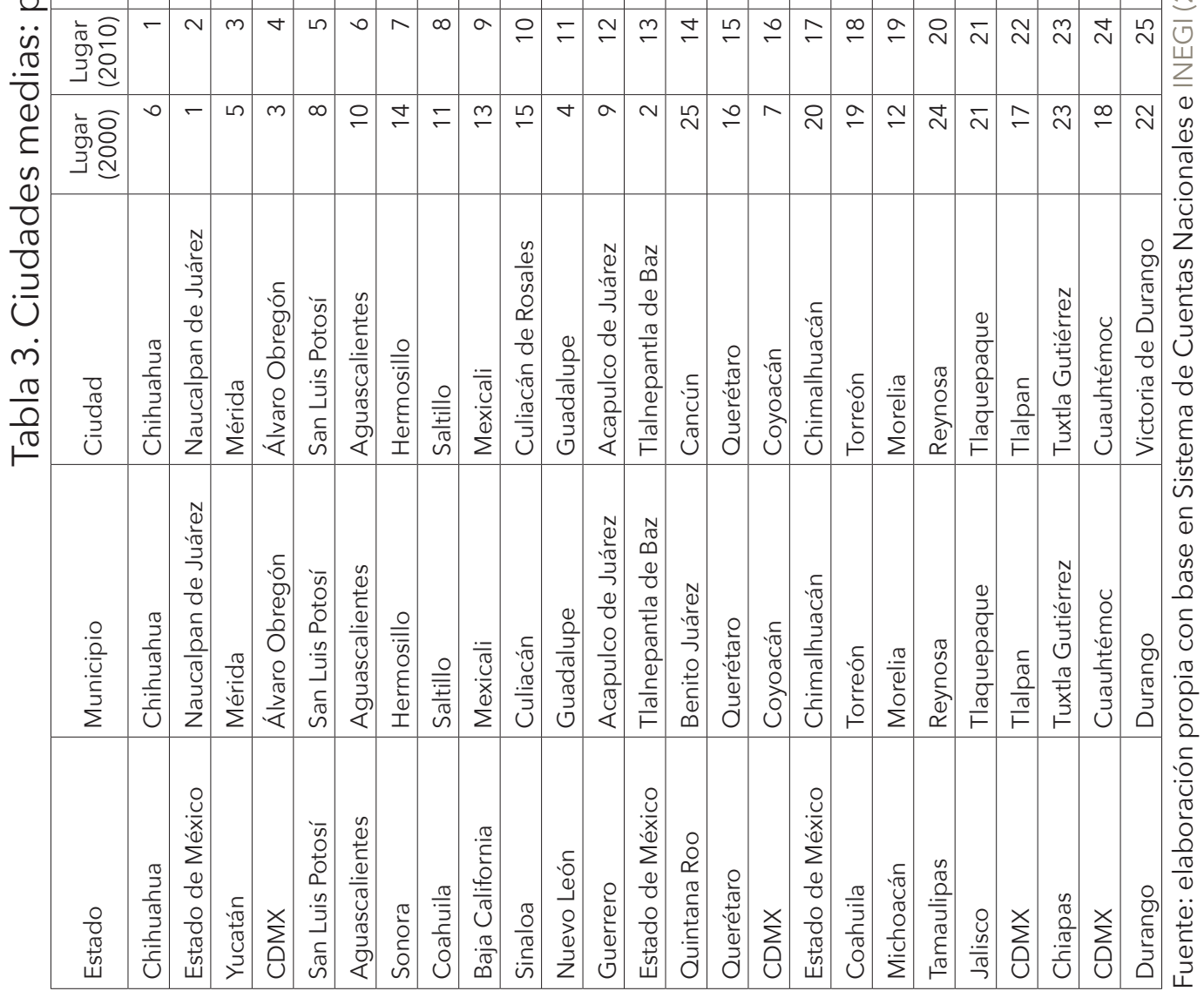
de pesos) y la de menor tamaño es Cancún, Quintana Roo (24 mil millones de pesos). Se observa una tendencia de las ciudades con mayor peso económico a concentrarse en Ciudad de México (CDMX).

Asimismo, la que registra el mayor nivel de desarrollo -medido por el PIB per cápita ( $P C$ ) - es Cuauhtémoc (CDMX), con 281 mil pesos promedio, y la de menor PIB per cápita de este grupo de ciudades es Mérida (Yucatán) (35 mil pesos). Por el nivel del PIB, 14 ciudades medias se ubican dentro de las 25 ciudades más importantes a escala nacional. De éstas, a excepción de Hermosillo (Sonora), Durango (Durango) y Culiacán (Sinaloa), todas son parte de áreas urbanas mayores estructuradas en zonas metropolitanas.

En resumen, si bien la participación del PIB de las ciudades medias en el PIB nacional es similar al peso de su población en el total nacional, la tasa de crecimiento económico registrada en los años 2000 y 2010 es mayor al de la población, sugiriendo una posición económica más central que la indicada por la población.

Por último, las ciudades medias analizadas se relacionan económica y socialmente con otros centros urbanos que determinan su dinámica y su posición central en la jerarquía urbana local, regional y nacional. En particular, la relevancia de las ciudades medias es más visible cuando se conectan con dos o más centros urbanos en otros municipios. Esto implica que su espacio urbano, sus funciones y sus actividades van más allá del límite municipal que originalmente las contenía (Consejo Nacional de Población [CONAPO], 2012). Las ciudades medias en zonas metropolitanas incorporan (o se incorporan) en su propia dinámica a municipios vecinos, manteniendo un alto grado de integración socioeconómica. En consecuencia, a mayor número de municipios conectados y entre más urbanos sean los municipios vecinos, mayor es la relevancia de la ciudad media central en la zona metropolitana. Así, cabe esperar que las ciudades medias delimitadas en zonas metropolitanas sean las de mayor efecto en la dinámica económica a escala nacional.

Lo anterior se basa en la idea de efectos de escala o de masa, entendidos como una de las ventajas elementales de una red de ciudades. Bajo este principio, un grupo de ciudades medias que interactúan en una red pueden alcanzar la misma dimensión funcional que una ciudad grande (Boix, 2003). Así, las ciudades medias interconectadas, principalmente en zonas metropolitanas, que funcionan a través de complementariedades y sinergias, pueden alcanzar una masa crítica para ofrecer funciones de mayor rango.

De este modo, en 2010 se tenían 59 zonas metropolitanas en todo el país que abarcaban un total de 367 municipios. En particular, el número de ciudades medias que se asentaron en zonas metropolitanas en 2010, es de 22. Estas zonas incluyen las ciudades de Aguascalientes, Mexicali, Torreón, Saltillo, Tuxtla Gutiérrez, Chihuahua, Coyoacán, Álvaro Obregón, Tlalpan, Cuauhtémoc, Chimalhuacán, Naucalpan, Tlalnepantla, Acapulco, Tlaquepaque, Morelia, Guadalupe, Querétaro, San Luis Potosí, Cancún, Reynosa y Mérida. Agrupan 21.1\% de la población urbana nacional y $22.9 \%$ del PIB nacional. La relevancia de las ciudades medias en zonas metropolitanas se refleja por el lugar que ocupan en la dinámica de las zonas. Así, $77 \%$ de este tipo de ciudades se consideran munici- 
pios centrales, y representan una fuerza socioeconómica de atracción de otros lugares de igual o menor jerarquía en el sistema urbano regional o nacional. En concreto, se vinculan directamente con 111 municipios, que representan una población de 61.5 millones de personas y un PIB total de 7.8 billones de pesos (11\% del PIB urbano total). Se observa una relevancia relativa de las ciudades medias agrupadas en zonas metropolitanas frente a las grandes.

En consecuencia, la integración de las primeras ciudades con vecinos con “mejores" características se refleja, por un lado, en su posición como ciudad media central regional, estatal o nacional y, por el otro, en su competitividad o capacidad de impulsar la actividad económica. Por lo que se espera que las ciudades medias tengan efectos en la dinámica nacional, en particular aquellas delimitadas o no en zonas metropolitanas - "con vecinos de calidad"-. Esto se comprueba de manera econométrica en la siguiente sección.

\section{Metodología y datos}

En esta sección se prueba de forma empírica la relación entre el tamaño de la ciudad y su relevancia económica, medida por el PIB, a nivel agregado. Una importante consecuencia de la urbanización es el cambio en la distribución del tamaño de las ciudades, derivada de la concentración geográfica de la producción en el interior de un país. Al respecto, dado los distintos tamaños de las ciudades, una parte de la tradición empírica urbana describe el tamaño de la distribución de las ciudades mediante la distribución de Pareto y considera un caso particular de esta distribución el conocido como la regla rango-tamaño (Gabaix y loannides, 2004). Esta regla establece que el producto del rango y la población de una ciudad es aproximadamente constante. De acuerdo con Soo (2005), cuando las ciudades se ordenan por el tamaño de su población y se regresa el logaritmo de su rango en función del logaritmo de su tamaño, se genera el coeficiente de Pareto, interpretado como una medida de concentración poblacional de las ciudades. Cuando este exponente es igual a 1, satisface las condiciones de la ley de Zipf. En términos matemáticos, esto representa la probabilidad de que el tamaño de una ciudad sea mayor a cierta $S$ es $P(s>S)=\alpha$ / $S \xi$, con $\xi \approx 1$ y a una constante positiva (Gabaix, 1999). En este sentido, Gabaix (1999) afirma que uno de los hechos estilizados acerca del tamaño relativo de las ciudades en economías de mercado, es el cumplimiento de la ley de Zipf.

De este modo, si bien parece aceptarse la relación natural entre los procesos de urbanización y de crecimiento económico, la evolución de la distribución del tamaño de las ciudades en el proceso de desarrollo económico dista mucho de ser directa. En consecuencia, puede preguntarse si una distribución del tamaño de las ciudades más equitativa favorece en efecto el desempeño económico de un país. En este sentido, tradicionalmente la relación negativa de la ley de Pareto entre los logaritmos del tamaño de la ciudad y el rango de la ciudad se considera en función del número de habitantes o de la extensión de la tierra; sin embargo, en este documento el tamaño de la ciudad se define considerando 
la magnitud de su economía ligada al tamaño de su población; esto es, el tamaño está determinado directamente por el PIB.

Por lo tanto, se mide la distribución del tamaño de las ciudades por el coeficiente de Pareto obtenido de una regresión auxiliar por lo general aceptada en la literatura. Para ello, primero se ordenan las ciudades por tamaño del PIB y se registra su ranking. Después se regresan los logaritmos de estos rangos con los logaritmos de sus tamaños económicos (PIB). En términos formales:

$$
\ln (\text { RangoPIB })=\alpha_{0}-\alpha_{1} \ln (\text { TamañoPIB })+\text { Error }
$$

El coeficiente estimado asociado con el logaritmo del tamaño (a1) es un estadístico que representa la distribución de tamaño de las ciudades. De acuerdo con Brañas y Alcalá (2014), si a1>1, la ciudad más grande es mayor que la predicción de la ley de Zipf, por ende, la desigualdad en el tamaño de las ciudades es mayor y aquella con el mayor tamaño ejerce un poder de dominación sobre las demás; si $0<a 1<1$, la ciudad más grande es menor que la predicción de la ley, lo que implica una distribución más homogénea del tamaño de las ciudades y un cierto equilibrio entre el poder económico de las mismas.

En este sentido, Berry y Okulicz (2012) señalan que la distribución del tamaño de las ciudades con $a 1=1$ puede considerarse como la distribución óptima que se observaría en la ausencia de fricciones u obstáculos al funcionamiento normal de la economía en el interior de un país. Una desviación del coeficiente de la unidad es consecuencia de choques externos, intervenciones gubernamentales o distorsiones en las decisiones de los agentes económicos que migran hacia ciudades al percibir mayores oportunidades o empresas que relocalizan su producción, lo que impulsa a estas regiones por arriba de otras de mayor tamaño. Estas desviaciones generan que las ciudades de menor rango tengan mayor peso económico agregado.

La forma de la distribución del tamaño de las ciudades fue propuesta inicialmente por Auerbach (1913), basado en la distribución de Pareto: $y=A \boldsymbol{x}^{-a}$, donde $x$ es la población de la ciudad; $y$, el rango de la ciudad, una vez ordenadas de mayor a menor tamaño; $A$, una constante positiva; y a, el exponente de Pareto. Una forma de estimar a es a través de una regresión de mínimos cuadrados ordinarios $(\mathrm{MCO})$ de los logaritmos rango-tamaño: $\log y=\log A$-alogx, donde $x$ representa el PIB de la $i$-ésima ciudad y, por ende, $a(\alpha>0)$ es una medida de la concentración de la actividad económica entre ciudades de diferentes tamaños. Los valores de $0<\alpha<1$ implicarían un tamaño de ciudades más equitativo.

Sin embargo, Gabaix e loannides (2004) argumentan que las estimaciones MCO son sesgadas en muestras pequeñas, lo que llevaría a considerar a las ciudades más grandes de lo que en realidad son. Este método subestima el valor verdadero del error estándar del exponente de Pareto, lo que conduce al rechazo del exponente. Proponen corregir el sesgo mediante un cambio de $1 / 2$ para que el rango sea óptimo. Así, el modelo formal es:

$$
\log (y-1 / 2)=\alpha_{0} \log A-\alpha_{1} \log x
$$


Además, Gabaix e Ibragimov (2012) muestran que bajo esta especificación el error estándar se distribuye asintóticamente como â' $(2 / n)^{1 / 2}$, donde $n$ es el tamaño de la muestra. La ecuación (2) se estima empleando MCO con errores estándar robustos que se prueban con el test de heterocedasticidad de Cook-Weisberg.

Para verificar la validez de la ley de Zipf, debe considerarse que la regla rango-tamaño representa esta ley cuando se considera sólo las grandes ciudades, pero esto no sucede necesariamente si se consideran todas las ciudades pues, en función de su definición, podrían incluirse localidades con al menos 2 mil 500 habitantes. En cualquier caso, en este documento se consideran centros urbanos de al menos 15 mil habitantes.

Como a es altamente sensible con la inclusión de toda la gama de ciudades (Fazio y Modica, 2015), se emplea el método de regresión de rangos móviles, útil cuando la variable dependiente es elástica a la variable independiente en diferentes segmentos, en el que se repite el proceso MCO, pero usando un punto de truncamiento menor que es, al mismo tiempo, el punto de partida para la siguiente submuestra. Este punto es el mismo y se fija en la ciudad más grande. El punto de truncamiento inferior disminuye en una ciudad con cada submuestra, por lo que el tamaño de ésta aumenta en uno. De este modo, se establece una submuestra para estimar con tamaño arbitrario de $\check{n}_{1}=20$, es decir, las primeras 20 ciudades con mayor PIB. La segunda submuestra es $\check{n}_{2}=$ $\check{n}_{1}+1=21$, que incluye las 21 ciudades más grandes; la tercera submuestra es $\check{n}_{3}=$ $\check{n}_{2}+1=22$, con las 22 ciudades mayores. El proceso continúa hasta incluir en la submuestra la ciudad más pequeña, de modo que éste termina representando la muestra total.

La muestra completa es de 575 ciudades con al menos 15 mil habitantes en 2010, que se ordenan de forma descendiente. Así, Iztapalapa (CDMX) resulta la ciudad de mayor tamaño y Tuxpan (Veracruz) la menor. Con este método se estiman 556(=575-20+1) coeficientes de Pareto al considerar ese número de regresiones a escala nacional.

Por último, la validación de la ley de Zipf no descansa en la aceptación o en el rechazo estadístico de $a_{1}=1$ en (1) y $a_{1}^{\prime}=1$ en (2), sino que se centra en la medida en que ésta se ajusta a los datos en lugar de su aceptación o rechazo estadístico (Peng, 2010). Para obtener resultados robustos se realizan las pruebas $t$ con hipótesis nula $a=1$. Se establece como hipótesis la existencia de un coeficiente de Pareto menor a la unidad como consecuencia del mayor peso económico relativo de las ciudades medias. La fuente de información es el Instituto Nacional de Estadística y Geografía (INEGI) y se consideran dos períodos para su comparación.

\section{Resultados}

Los resultados de las estimaciones de las ecuaciones (1) y (2) se presentan en la tabla 4. Las dos especificaciones tienen una evolución similar en la que se aprecia una tendencia decreciente de a y $a^{\prime}$, pero siempre en el intervalo 
$0<a_{1}<1$. También, como se esperaba, se mantiene $a<a^{\prime}$. Así, se acepta que el peso económico de la ciudad más grande es menor que la predicción de la ley, lo que implica una distribución más homogénea del tamaño de las ciudades y un cierto equilibrio entre el poder de las mismas. En otras palabras, el resultado refleja la mayor relevancia de las ciudades de menor tamaño en el PIB nacional.

Sobre el año 2000, se tiene que el valor promedio del exponente de Pareto (a) en todas las regresiones es cercano a 0.681 , mientras que el valor de este exponente en la muestra completa (575 ciudades) es 0.489 . Al respecto del exponente $a^{\prime}$ los valores respectivos son 0.793 y 0.499 . Así, todos los exponentes estimados por (1) son inferiores a 1 y sólo dos son mayores a la unidad cuando se estiman por (2). Esta situación se replica en el caso del año 2010, donde el valor promedio del exponente a es cercano a 0.709 y el de la muestra completa es 0.506 , al tiempo que los valores del exponente $a^{\prime}$ son 0.835 y 0.525 . En general, se tiene que la ley de Zipf parece no cumplirse en las ciudades mexicanas considerando su tamaño económico. Un resultado similar se encuentra en Pérez, Guzmán y Angulo (2015), Soo (2005) y Urzúa (2001). ${ }^{4}$

En concreto, cuando se consideran las submuestras estimadas con entre 20 y 25 ciudades, los exponentes de Pareto ajustados $\left(a^{\prime}\right)$ son 1.044 y 1.018 , respectivamente, para 2000, y 1.099 y 1.018 para 2010. Esto indica que la ciudad más grande (Iztapalapa, CDMX) tiene mayor peso relativo que lo señalado por la ley de Zipf en esos años con esas submuestras. Esto se traduce en una mayor desigualdad en el tamaño económico de las ciudades.

No obstante, la desigualdad se reduce conforme las submuestras aumentan de tamaño; el coeficiente de Pareto estimado disminuye por debajo de la unidad. El valor estimado de $\alpha^{\prime}$ es monótonamente decreciente para todas las submuestras. ${ }^{5}$ Así, desde el punto de truncamiento de 30 y 40 ciudades cada año, el exponente muestra un valor menor a la unidad, lo que implica una distribución más homogénea del tamaño económico de las ciudades y, a partir de este punto, un cierto equilibrio entre el poder de las mismas, esto es, un poder relativamente similar entre las ciudades a pesar de sus diferentes tamaños.

Los errores estándar se corrigen por el método propuesto por Gabaix e Ibragimov (2012) y pasan el test de heterocedasticidad de Cook-Weisberg. En relación con 2000 , las estimaciones son significativas hasta la submuestra de tamaño 300 que incluye las 25 ciudades medias. En ésta, se registra una distribución desigual en términos relativos del poder económico de las ciudades; en contraste, en la primera submuestra (20 ciudades), el $\alpha^{\prime}>1$, e incluye siete ciudades medias. Aún más, en la submuestra de tamaño $n=50$ con $a_{1}>1$ se incluyen 18 ciudades medias (más de $80 \%$ de este tipo de ciudades) y si se considera la submuestra $n=100$; también con $a_{1}>1$ se tendría que $96 \%$ de las ciudades medias están dentro de un sistema urbano con una distribución del tamaño económico equitativa.

4 En contraste, Benita y Martínez (2011) encuentran evidencia favorable para la ley de Zipf al considerar la estructura industrial en México relativa a los años 1999, 2004 y 2009. Así mismo, Lugo (2009) valida la ley para la distribución del sistema metropolitano en México entre 1990 y 2005.

5 Cabe señalar que para las submuestras de mayor tamaño el valor del exponente de Pareto disminuye. De hecho, desde la submuestra de tamaño 200 se tiene un grado de dispersión excesivo (a1<0.7), de acuerdo con lo señalado por Brañas y Alcalá (2014). 


\begin{tabular}{|c|c|c|c|c|c|c|c|c|c|c|c|c|c|c|c|c|c|c|}
\hline \multirow{8}{*}{\multicolumn{2}{|c|}{$\stackrel{\circ}{2}$}} & $\underset{\mathrm{U}}{E}$ & L & $\wedge$ & $\infty$ & $\stackrel{ }{-}$ & $\stackrel{\circ}{-}$ & $\simeq$ & $\stackrel{ \pm}{\square}$ & $\stackrel{\infty}{\leftarrow}$ & $\stackrel{\sim}{\sim}$ & $\stackrel{\llcorner}{\sim}$ & $\stackrel{\sim}{\sim}$ & $\stackrel{\llcorner}{\sim}$ & $\stackrel{\llcorner}{\sim}$ & $\stackrel{\llcorner}{\sim}$ & $\stackrel{\llcorner}{\sim}$ & \\
\hline & & 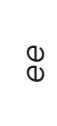 & 寺 & $\begin{array}{l}\text { r } \\
\text { Oे } \\
\text { r. }\end{array}$ & 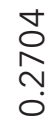 & $\begin{array}{l}\stackrel{m}{m} \\
\stackrel{+}{+} \\
0\end{array}$ & 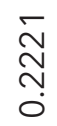 & $\frac{o}{a}$ & $\begin{array}{l}0 \\
\frac{0}{0} \\
0\end{array}$ & 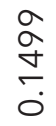 & $\frac{\infty}{\infty}$ & $\begin{array}{l}0 \\
0 \\
0 \\
0 \\
0\end{array}$ & \begin{tabular}{l}
$\hat{\jmath}$ \\
\multirow{0}{0}{} \\
0
\end{tabular} & $\begin{array}{l}\text { O } \\
\text { + } \\
\text { ○. }\end{array}$ & $\begin{array}{l}\text { ๖े } \\
\text { गे } \\
0\end{array}$ & $\begin{array}{l}\frac{\omega}{m} \\
\stackrel{0}{0} \\
0\end{array}$ & I & \\
\hline & & & 粪 & 类 & 粪 & 絭 & 粪 & 粪 & 娄 & 粪 & 絭 & ${ }_{*}^{*}$ & * & & & & & \\
\hline & & ס' & $\begin{array}{l}0 \\
\infty \\
\circ \\
0 \\
\vdots \\
r\end{array}$ & 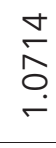 & 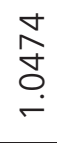 & $\stackrel{\stackrel{\infty}{N}}{\frac{\infty}{\sigma}}$ & $\begin{array}{l}m \\
m \\
a \\
\vdots \\
0\end{array}$ & $\begin{array}{l}0 \\
\infty \\
10 \\
\alpha \\
0 \\
0\end{array}$ & $\begin{array}{l}\stackrel{o}{o} \\
\frac{a}{a} \\
\dot{0}\end{array}$ & $\begin{array}{l}- \\
\infty \\
\infty \\
\infty \\
0\end{array}$ & \begin{tabular}{l}
$\infty$ \\
0 \\
\multirow{+}{0}{} \\
$\infty$ \\
0 \\
0
\end{tabular} & $\begin{array}{l}\overline{0} \\
0 \\
0 \\
0\end{array}$ & $\begin{array}{l}\infty \\
m \\
\infty \\
\stackrel{\infty}{n} \\
0\end{array}$ & 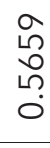 & 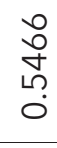 & $\begin{array}{l}\stackrel{\infty}{+} \\
\stackrel{N}{ஸ} \\
\stackrel{0}{0}\end{array}$ & $\begin{array}{l}\text { O } \\
\stackrel{1}{n} \\
\infty \\
0 \\
0\end{array}$ & 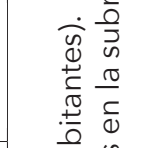 \\
\hline & & 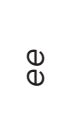 & \begin{tabular}{l}
0 \\
$\infty$ \\
$\infty$ \\
\multirow{2}{0}{} \\
0
\end{tabular} & 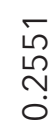 & 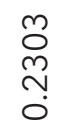 & 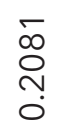 & $\frac{\infty}{\stackrel{\infty}{~}}$ & $\begin{array}{l}\stackrel{0}{2} \\
\stackrel{+}{+} \\
\dot{0}\end{array}$ & 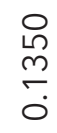 & 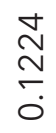 & $\begin{array}{l}\infty \\
2 \\
0 \\
0 \\
0 \\
0\end{array}$ & $\begin{array}{l}\bar{\infty} \\
\stackrel{0}{\circ} \\
0\end{array}$ & $\begin{array}{l}0 \\
0 \\
0 \\
0 \\
0\end{array}$ & $\begin{array}{l}\stackrel{8}{0} \\
\text { m. } \\
\text { o. }\end{array}$ & $\begin{array}{l}\stackrel{0}{m} \\
\text { o } \\
\dot{0}\end{array}$ & $\begin{array}{l}\text { ठ } \\
\text { } \\
\text { o } \\
\text { o. }\end{array}$ & 1 & 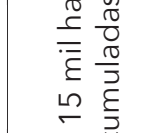 \\
\hline & & & 类 & 类 & 絭 & 粪 & 絭 & 类 & 絭 & 絭 & 类 & * & * & & & & & \\
\hline & & 0 & $\begin{array}{l}\hat{m} \\
\grave{a} \\
\vdots\end{array}$ & $\begin{array}{l}\frac{\infty}{\circ} \\
\vdots \\
\vdots\end{array}$ & $\begin{array}{l}\bar{\alpha} \\
\infty \\
0 \\
0\end{array}$ & $\begin{array}{l}\stackrel{2}{0} \\
\stackrel{0}{\infty} \\
0 \\
0\end{array}$ & $\begin{array}{l}m \\
0 \\
0 \\
0 \\
0 \\
0\end{array}$ & \begin{tabular}{l}
$\stackrel{a}{\gamma}$ \\
\multirow{2}{*}{} \\
0
\end{tabular} & $\begin{array}{l}m \\
\stackrel{m}{m} \\
\stackrel{0}{0}\end{array}$ & 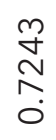 & 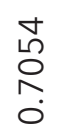 & $\begin{array}{l}0 \\
0 \\
0 \\
\text { ?. } \\
0\end{array}$ & $\begin{array}{l}\stackrel{\infty}{+} \\
\stackrel{+}{\Lambda} \\
0\end{array}$ & 을 & 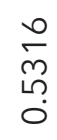 & 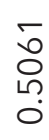 & $\begin{array}{l}\stackrel{8}{\circ} \\
\stackrel{0}{0} \\
0\end{array}$ & 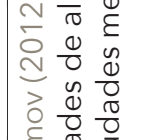 \\
\hline & & c & $\stackrel{৩}{\sim}$ & $\stackrel{\llcorner}{\sim}$ & ஓ & $\stackrel{\varphi}{m}$ & 우 & 유 & ○ & $\stackrel{\circ}{\wedge}$ & 음 & ㅇ & ৪্ & O & 음 & $\begin{array}{l}\sqrt{\pi} \\
\stackrel{0}{0}\end{array}$ & $\begin{array}{l}\frac{0}{0} \\
0 \\
\frac{1}{\varepsilon} \\
0 \\
0 \\
0\end{array}$ & 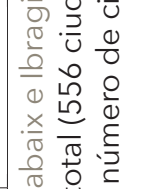 \\
\hline \multirow{8}{*}{\multicolumn{2}{|c|}{ 유 }} & $\underset{\Xi}{E}$ & $\wedge$ & $\wedge$ & $\infty$ & $\mp$ & $\underset{\leftarrow}{\mp}$ & $\stackrel{\infty}{\sim}$ & $\stackrel{\ominus}{\sim}$ & $\stackrel{\sim}{v}$ & $\stackrel{d}{\sim}$ & $\stackrel{\llcorner}{N}$ & $\stackrel{\llcorner}{\sim}$ & $\stackrel{\llcorner}{\sim}$ & $\stackrel{\llcorner}{\sim}$ & $\stackrel{\stackrel{n}{N}}{\sim}$ & $\stackrel{\llcorner}{\sim}$ & \multirow{8}{*}{ 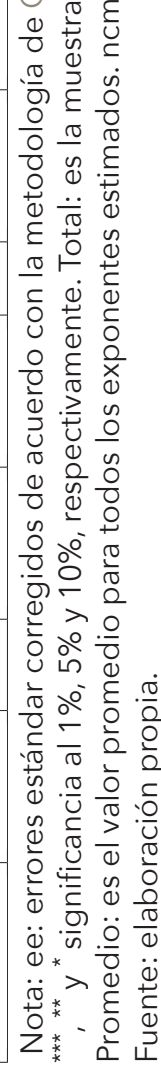 } \\
\hline & & 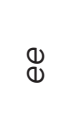 & 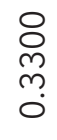 & 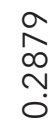 & $\begin{array}{l}\stackrel{a}{0} \\
\stackrel{2}{n} \\
\stackrel{0}{0}\end{array}$ & 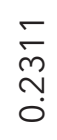 & $\frac{O}{\bar{N}}$ & $\begin{array}{c}\stackrel{0}{N} \\
\infty \\
\vdots \\
\vdots\end{array}$ & $\begin{array}{l}\infty \\
\infty \\
\stackrel{1}{ก} \\
\leftarrow \\
0\end{array}$ & $\frac{\stackrel{\sim}{\sim}}{\stackrel{+}{*}}$ & 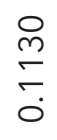 & $\begin{array}{l}m \\
m \\
0 \\
0 \\
0\end{array}$ & 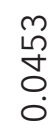 & $\begin{array}{l}\stackrel{0}{\infty} \\
\text { ñ } \\
0 \\
\dot{0}\end{array}$ & $\begin{array}{l}\stackrel{\infty}{N} \\
\tilde{m} \\
0 \\
0\end{array}$ & $\begin{array}{l}\text { aे } \\
\text { ปे } \\
\text { o. }\end{array}$ & I & \\
\hline & & & 类 & 类 & 絭 & 絭 & 絭 & 絭 & 絭 & 类 & 类 & ${ }_{*}^{*}$ & * & & & & & \\
\hline & & ס & $\begin{array}{l}\hat{m} \\
\text { J } \\
\stackrel{-}{r}\end{array}$ & 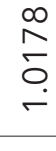 & $\begin{array}{l}\circ \\
\text { 으 } \\
2 \\
\vdots \\
0\end{array}$ & $\begin{array}{l}a \\
0 \\
0 \\
a \\
0\end{array}$ & 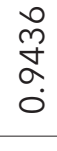 & $\begin{array}{l}\overline{0} \\
\vdots \\
\vdots \\
0\end{array}$ & $\begin{array}{l}0 \\
\infty \\
0 \\
\infty \\
0 \\
0\end{array}$ & 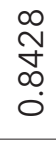 & 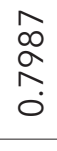 & $\begin{array}{l}\stackrel{\infty}{N} \\
\tilde{N} \\
0 \\
0\end{array}$ & 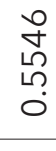 & 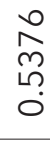 & $\begin{array}{l}m \\
2 \\
\frac{2}{2} \\
0\end{array}$ & 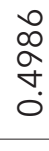 & $\begin{array}{l}m \\
m \\
\frac{m}{2} \\
0\end{array}$ & \\
\hline & & 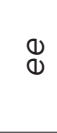 & 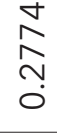 & $\underset{\substack{f \\
\sim}}{\stackrel{\sim}{0}}$ & $\underset{\check{N}}{\stackrel{\Sigma}{0}}$ & $\frac{\infty}{2} \frac{a}{a}$ & $\begin{array}{l}\stackrel{0}{0} \\
\stackrel{0}{0}\end{array}$ & $\frac{\stackrel{p}{p}}{\leftarrow}$ & $\frac{2}{2}$ & $\frac{\stackrel{L}{N}}{\stackrel{5}{\sigma}}$ & 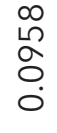 & $\begin{array}{l}\infty \\
\stackrel{1}{n} \\
\\
0 \\
0\end{array}$ & 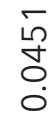 & \begin{tabular}{l}
\multirow{N}{N}{} \\
m \\
$\dot{0}$
\end{tabular} & $\begin{array}{l}\stackrel{m}{N} \\
\stackrel{m}{0} \\
\dot{0}\end{array}$ & 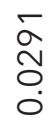 & I & \\
\hline & & & $\begin{array}{l}* \\
k \\
k\end{array}$ & 类 & 絭 & 粪 & 类 & 粪 & 类 & 类 & 类 & $\stackrel{*}{*}$ & * & & & & & \\
\hline & & 0 & $\begin{array}{l}\bar{N} \\
\hat{\infty} \\
0 \\
0\end{array}$ & $\begin{array}{l}1 \\
\text { ம } \\
\infty \\
\infty \\
0\end{array}$ & $\begin{array}{l}\text { Jे } \\
\text { மे } \\
\infty \\
0 \\
0\end{array}$ & $\begin{array}{l}\hat{n} \\
\stackrel{2}{0} \\
\infty \\
0\end{array}$ & $\begin{array}{l}\stackrel{L}{n} \\
\stackrel{n}{N} \\
0\end{array}$ & $\begin{array}{l}0 \\
\infty \\
\\
0\end{array}$ & $\begin{array}{l}\hat{a} \\
\stackrel{0}{0} \\
0\end{array}$ & $\begin{array}{l}m \\
\text { ñ } \\
0 \\
0 \\
0\end{array}$ & $\begin{array}{l}\overline{\hat{N}} \\
\text { o } \\
0\end{array}$ & 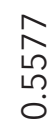 & $\begin{array}{l}\infty \\
\stackrel{\infty}{n} \\
0 \\
0\end{array}$ & $\begin{array}{l}\text { مे } \\
\text { N̦ } \\
\text { ?. }\end{array}$ & 官 & 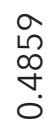 & $\begin{array}{l}0 \\
0 \\
0 \\
0 \\
0\end{array}$ & \\
\hline & & $c$ & i & $\stackrel{\llcorner}{\sim}$ & ஓ & $\stackrel{\llcorner}{m}$ & 암 & 요 & ○ & $\stackrel{\circ}{\wedge}$ & 으 & ঃ & ஓ্ & 우 & 움 & $\begin{array}{l}\text { त्ञ } \\
\stackrel{0}{0}\end{array}$ & $\begin{array}{l}-\frac{.}{0} \\
0 \\
\text { है } \\
0 \\
0\end{array}$ & \\
\hline
\end{tabular}


Con respecto al año 2010, las estimaciones son significativas hasta la submuestra de tamaño 300, que también incluye las 25 ciudades medias, y se registra una distribución relativamente desigual del poder económico de las ciudades; en contraste, en la primera submuestra (20 ciudades), el $\alpha^{\prime}>1$, e incluye cinco ciudades medias. En la submuestra de tamaño $n=50$ con $a_{1}>1$ se incluyen 12 ciudades medias y en la sub-muestra $n=100$, también con $a_{1}>1$, se tiene que $92 \%$ de las ciudades medias están dentro de este sistema urbano que puede considerarse equitativo en términos relativos. Así, parece aceptarse que el poder económico de las ciudades medias es relativamente homogéneo al de las ciudades de mayor tamaño en la submuestra.

Figura 1. Logaritmo del tamaño versus logaritmo del rango. Muestra completa
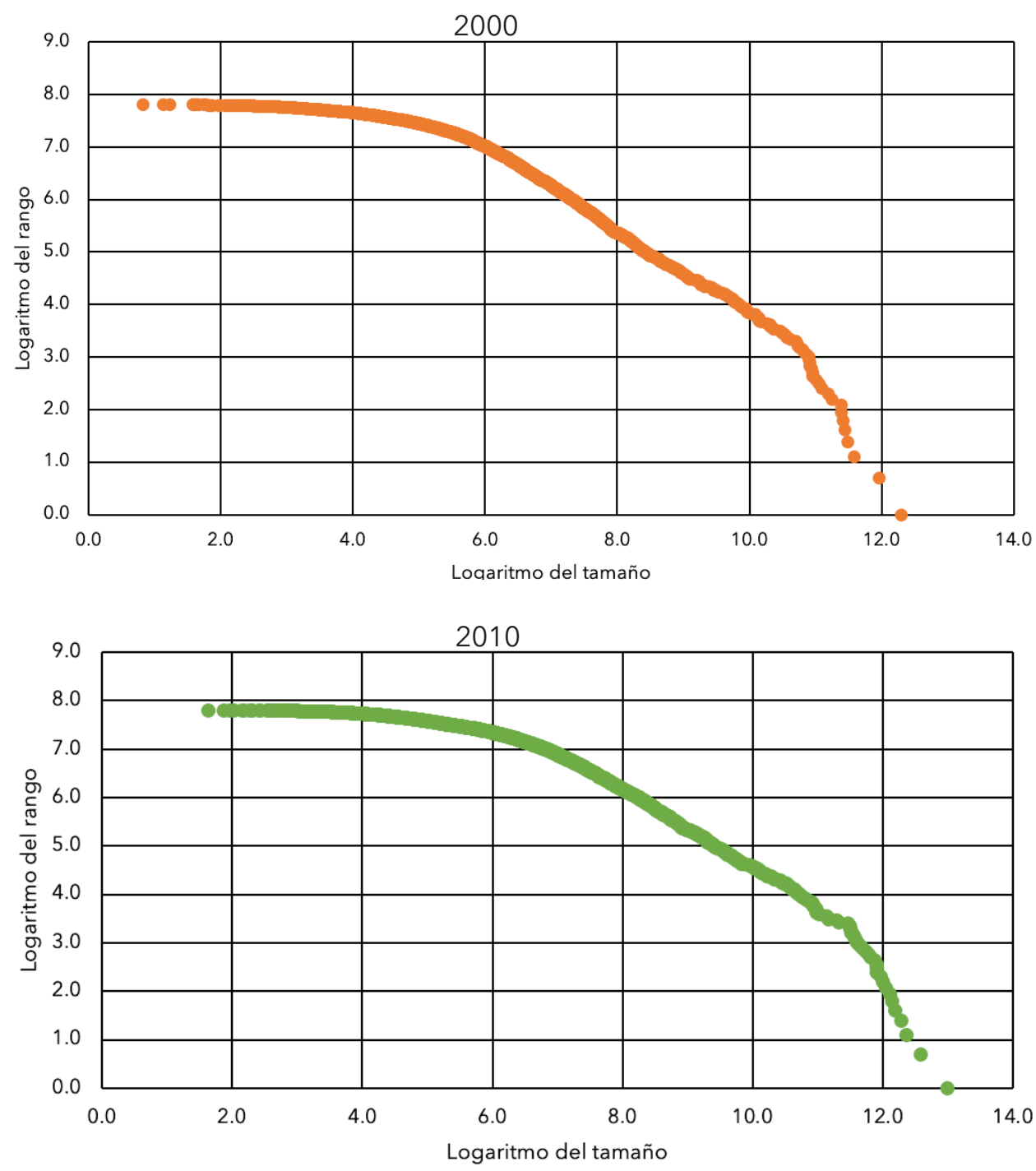

Fuente: elaboración propia. 
La relevancia de las ciudades medias puede apreciarse en la figura 1, que muestra la dispersión del logaritmo del rango contra el logaritmo del tamaño económico (PIB) de las ciudades para la muestra completa. El diagrama no refleja una línea recta sino cóncava, lo que está de acuerdo con el hecho de que el exponente de Pareto varía en función del tamaño de la submuestra. La concavidad sugiere que las ciudades más grandes son demasiado pequeñas y las ciudades de menor tamaño están subrepresentadas. Rossi y Wright (2007) enfatizan esta regularidad empírica. La concavidad registrada sugiere la desviación de la ley de Zipf, lo que es evidencia de distorsiones del sistema urbano, consecuencia posiblemente de aglomeraciones de menor tamaño con mayor contribución a la dinámica económica, si bien con menores niveles de eficiencia por representar menores economías de urbanización asociadas con cuestiones institucionales, de localización e históricas (Soo, 2005).

\section{Conclusiones}

Una de las regularidades más importantes con respecto a la ubicación geográfica de la actividad económica en el interior de los países es la concentración en las ciudades. En este sentido, en el documento se analizó la relevancia de las ciudades medias en México durante los años 2000 y 2010, para los que existe información, a escala de localidad, sobre las variables de población y PIB, lo que permite determinar las áreas urbanas de interés y su actividad socioeconómica. Se identificaron 25 ciudades con una población de entre 500 mil y 1 millón de habitantes en 2010, consideradas ciudades medias. Esas aglomeraciones concentran cerca de una de cada cuatro personas en el país y un peso de cada cuatro del PIB nacional. Se determinó la importancia de las ciudades medias en la actividad económica nacional, a partir de la metodología planteada por la ley de Zipf, que mide la distribución del tamaño, en este caso económico, de las ciudades por el coeficiente de Pareto.

Para ello se realizaron estimaciones en 556 submuestras de dos años y dos especificaciones distintas. Los resultados permiten aceptar la hipótesis de un coeficiente de Pareto menor a la unidad para submuestras de mayor tamaño. Se argumenta que esto es consecuencia del mayor peso económico relativo de las ciudades medias. En efecto, el comportamiento de las ciudades medias representa un mecanismo subyacente que impulsa la actividad económica a escala nacional.

Esto implica que la distribución relativamente homogénea entre el tamaño económico de las ciudades favorece la actividad económica en centros urbanos de tamaño medio, así como en los municipios con los que se vinculan, sobre todo aquellos en zonas metropolitanas, y en la región en la que se desenvuelven $y$, en algún grado, en el total nacional. Las distintas conexiones de las ciudades medias hacia adelante -con ciudades de mayor jerarquía- y hacia atrás -con ciudades menores y lugares rurales- representan el potencial de las mismas como puentes económicos. 
Dado este mecanismo promotor de la economía, los hacedores de política deben implementar políticas regionales y urbanas que favorezcan su dinámica interna, así como sus vínculos con aglomeraciones urbanas menores o mayores, según sea el caso, en un contexto de relaciones entre núcleo y periferia. En términos simples, las políticas deben fomentar la aglomeración económica en estas ciudades, lo que se asocia con el papel de éstas en la jerarquía urbana, los activos centrales de cada una y la conectividad - física o no- con otros lugares dentro del sistema urbano regional y nacional. Las políticas de fomento de las ciudades deben recordar que el papel socioeconómico de las ciudades medias deriva justamente de su posición intermedia entre las grandes urbes y las ciudades de menor tamaño y zonas rurales cercanas.

Por último, se reconoce la importancia de considerar otros estudios para comprender con mayor detalle el rol de las ciudades medias mexicanas, entre otros: evaluación de los vínculos directos con el ordenamiento territorial, reducción de la pobreza y de la desigualdad, la contención de la migración a grandes ciudades y la capacidad de innovación para integrarse a la economía del conocimiento. De igual manera, las ciudades podrían clasificarse por subgrupos en función de distintas características, como la distancia con zonas metropolitanas, y estudiar las diferencias de los intergrupos.

\section{Referencias}

Acs, Z., Desai, S., y Hessels, J. (2008). Entrepreneurship, economic development and institutions. Small Business Economics, 31, 219-234.

Álvarez, G. (2017). Morfología y estructura urbana en las ciudades medias mexicanas. región y sociedad, 29(68), 153-191. doi: 10.22198/rys.2017.68.a872

ATKearney. (2017). Global Cities 2017: Leaders in a World of Disruptive Innovation. Seúl: A. T. Kearney.

Audretsch, D., Belitski, M., y Desai, S. (2015). Entrepreneurship and economic development in cities. Annales of Regional Science, 55: 33-60. doi: 10.1007/ s00168-015-0685-x

Bajpai Jitendra, N., y Muzzini, E. (2016). Cities as Growth Accelerators: Fostering National and Urban Development Policies for Success. Caracas: Corporación Andina de Fomento.

Banco Mundial (BM). (2015). East Asia's Changing Urban Landscape: Measuring a Decade of Spatial Growth. Washington: Banco Mundial.

Benita, F., y Martínez, J. (2011). Regularidades empíricas de la estructura industrial mexicana: evidencia de la ley de Zipf. Investigación y Ciencia, 52, 21-26.

Berdegué, J., y Soloaga, I. (2018). Small and medium cities and development of Mexican rural areas. World Development, 107, 277-288.

Berry, B., y Okulicz, A. (2012). The city size distribution debate: resolution for US urban regions and megalopolitan areas. Cities, 29(1), 17-23. doi: $10.1016 /$ j.cities.2011.11.007 
Boix, R. (2003). Networks of Cities and Growth: Theory, Network Identification and Measurement of the Network Externality. Florencia: Universidad de Florencia.

Bolay, J., y Ravinovich, A. (2004). Villes intermédiaires en Amérique Latine. Risques et potentiels pour un développement urbain cohérent. Seminario internacional Medium-Sized Cities and Globalization: Renewing the Analysis and Strategies. Montreal: Universidad de Montreal.

Bourne, S. (1982). Internal Structure of the City. Nueva York: Oxford University Press.

Brañas, P., y Alcalá, F. (2014). Entropía, aglomeración urbana y la Ley del 1: evidencia para las regiones españolas. Gran Canaria: XVII Encuentro de Economía Aplicada.

Cadena, A., Remes, J., y Manyika, J. (2011). Building globally competitive cities: the key to Latin American growth. Nueva York: McKinsey Global Institute.

Camagni, R., Capello, R., y Caragliu, A. (2013). One or infinite optimal city sizes? In search of an equilibrium size for cities. The Annals of Regional Science, 51(2), 309-341.

Cheshire, P., Magrini, S., Medda, F., y Monastiriotis, V. (2004). Cities are not isolated states. Research Papers in Environment and Spatial Analysis Series no. 91. Londres: London School of Economics and Political Science.

Clark Hon, G., y Clark, G. (2014). Nations and the Wealth of Cities: a New Phase of Urban Policy. Londres: Centre for London.

Consejo Nacional de Población (CONAPO). (2012). Delimitación de las zonas metropolitanas en México 2010. Ciudad de México: Consejo Nacional de Población.

Core Cities. (2013). Competitive cities, prosperous people: a core cities prospectus for growth. Recuperado de https://www.corecities.com/sites/default / files / field/attachment/Competitive\%20Cities\%2C\%20Prosperous\%20 People_\%20Final\%20Draft.pdf

Cox, E., y Longlands, S. (2016). The Role of Small and Medium-Sized Towns and Cities in Growing the Northern Powerhouse. Manchester: IPPR North.

Dahiya, B. (2012). Cities in Asia, 2012: Demographics, economics, poverty, environment and governance. Cities, 29, S44-S61.

Dijkstra, L., Garcilazo, E., y McCann, P. (2013). The economic performance of European cities and city regions: myths and realities. European Planning Studies, 21(3), 334-354. doi: 10.1080/09654313.2012.716245

Duranton, G. (2015). Growing through Cities in Developing Countries. Oxford: Oxford University Press.

Duroudier, S. (2014). The social and spatial divisions of intermediate cities in the United States. L'Espace Géographique, 43, 134-147. doi: 10.3917/ eg.432.0134

Fang, C., Pang, B., y Liu, H. (2017). Global city size hierarchy: spatial patterns, regional features, and implications for China. Habitat International, 66, 149-162. doi: 10.1016/j.habitatint.2017.06.002 
Fazio, G., y Modica, M. (2015). Pareto or log-normal? Best fit and truncation in the distribution of all cities. Journal of Regional Science, 55(5), 736-756. doi: $10.1111 /$ jors. 12205

Florida, R. (2002). The Rise of the Creative Class: And How it's Transforming Work, Leisure, Community and Everyday Life. Nueva York: Basic Books.

Fritsch, M., y Mueller, P. (2004). Effects of new business formation on regional development over time. Regional Studies, 38, 961-975.

Gabaix, X. (1999). Zipf's Law for cities: an explanation. Quarterly Journal of Economics, 114, 739-767.

Gabaix, X., e Ibragimov, R. (2012). Rank -1/2: a simple way to improve the OLS estimation of tail exponentes. Journal of Business and Economic Statistics, 29(1), 24-39.

Gabaix, X., e loannides, Y. (2004). The evolution of city size distribution. Handbook of Regional and Urban Economics, 4, 2431-2378.

Giesen, K., y Suedekum, J. (2009). Zipf's law for cities in the regions and the country. IZA DP, 3928.

Glaeser, E. (2011). The Triumph of the City: How our Greatest Invention Makes US Richer, Smarter, Greener, Healthier and Happier. Nueva York: Penguin Press.

González, A., y Gallegos, G. (2014). El producto interno bruto de los municipios de México: II. Estados M-Z. Revista Mexicana de Ciencias Agrícolas, 5(8), 1405-1421.

Grabher, G. (2018). Marginality as strategy: leveraging peripherality for creativity. Environment and Planning A, 50(8), 1785-1794. doi: 10.1177/0308518X18784021

Guerrero, P., Lucenti, K., y Galarza, S. (2009). Trade logistics and regional integration in Latin America and the Carribean. IADB Working Papers 148.

Henderson, J. (2000). The effects of urban concentration on economic growth. National Bureau of Economic Research. Working Paper 7503.

Henderson, J., y Thisse, J. (eds.). (2004). Handbook of Regional and Urban Economics, Cities and Geography. Amsterdam: Elsevier.

Hiernaux, D., y Lindón, A. (2004). Desterritorialización y reterritorialziación metropolitana: la Ciudad de México. Documents d'Anàlisi Geogràfica, 44:7178.

Instituto Nacional de Estadística y Geografía. (2000). XII Censo General de Población y Vivienda 2000. Recuperado de https://www.inegi.org.mx/programas/ccpv/2000/default.html?ps=microdatos

Instituto Nacional de Estadística y Geografía. (2010). Censo de Población y Vivienda 2010. Recuperado de https://www.inegi.org.mx/programas/ ccpv/2010/

Jones, N., Bhatta, B., Gill, G., Pantuliano, S., Singh, H., Timsina, D., Uppadhaya, S., y Walker, D. (2009). Governance and Citizenship from Below: Views of Poor and Excluded Groups and their Vision for a New Nepal, ODI Working Paper 301.

Katz, B., y Bradley, J. (2013). The Metropolitan revolution: How Cities and Metros are Fixing our Broken Politics and Fragile Economy. Filadelfia: Brookings Institution Press. 
Khanna, P. (2016). Connectography: Mapping the Future of Global Civilization. Nueva York: Random House.

Klaufus, C. (2010). Watching the city grow: remittances and sprawl in intermediate Central American cities. Environment and Urbanization, 22(1), 125137. doi: 10.1177/0956247809359646

Krugman, P. (1991). Geography and Trade. Cambridge: The MIT Press.

Lugo Olmos, I. (2009). Distribución y crecimiento urbano en el sistema metropolitano de México: un enfoque de sistemas complejos (tesis de Doctorado en Economía). Recuperada de http://catarina.udlap.mx/u_dl_a/tales/documentos/dec/lugo_o_i/

McCann, P., y Acs, Z. (2011). Globalization: countries, cities and multinationals. Regional Studies, 45(1), 17-32. doi: 10.1080/00343404.2010.505915

McFarland, C. (2017). Local Economic Conditions 2017. Research and Analysis of Local Economies: Going beyond "Urban vs. Rural". Washington: National League of Cities.

Oliveras, J., y Cartanyá, A. (2015). Ciudades difusas y confusas, conurbaciones y áreas metropolitanas. El caso del camp de Tarragona. International Conference on Regional Science. Terragona, España: Universidad Rovira i Virgili.

Organización para la Cooperación y Desarrollo Económicos (OCDE). (2006). Competitive Cities in the Global Economy. París: Organización para la Cooperación y Desarrollo Económicos.

Organización de las Naciones Unidas (ONU)-Habitat. (2010). The state of Asian cities 2010/11. Conferencia de las Naciones Unidas Sobre Vivienda y Desarrollo Urbano Sostenible: Fukuoka.

Parr, J., y Jones, C. (1983). City size distributions and urban density functions: some interrelationships. Journal of Regional Science, 23(3), 283-307. doi: 10.1111/j.1467-9787.1983.tb00991.x

Peng, G. (2010). Zipf's law for Chinese cities: rolling sample regressions. Physica A, 389, 3804-3813. doi: 10.1016/j.physa.2010.05.004

Pérez, E., Guzmán, L., y Angulo, F. (2015). Distributions of city sizes in Mexico during the 20th century. Chaos, Solutions \& Fractals, 73, 64-70. doi: 10.1016/j.chaos.2014.12.015

Pike, A., Rodriguez, A., y Tomaney, J. (2006). Local and Regional Development. Londres: Routledge.

Puissant, S., y Lacour, C. (2011). Mid-sized French cities and their niche competitiveness. Cities, 28, 433-443. doi: 10.1016/j.cities.2011.05.008

Rosen, K., y Resnick, M. (1980). The size distribution of cities: an examination of the Pareto law and primacy. Journal of Urban Economics, 8(2), 165-186. doi: 10.1016/0094-1190(80)90043-1

Rosenthal, S., y Strange, W. (2004). Evidence on the nature and sources of agglomeration economies. En J. Henderson y J. Thiss (eds.). Handbook of Regional and Urban Economics, Cities and Geography (pp. 2119-2171). Amsterdam: Elsevier.

Rossi, E., y Wright, M. (2007). Urban structure and growth. Review of Economic Studies, 74(2), 597-624. 
Sridhar, K., y Wan, G. (2010). Firm location choice in cities: evidence from China, India, and Brazil. China Economic Review, 21, 113-122. doi: 10.1016/j. chieco.2009.11.003

Sobrino, J. (1999). Desarrollo urbano en México a partir de 1980. Toluca: El Colegio Mexiquense.

Soo, K. (2005). Zipf's Law for cities: a cross-country investigation. Regional Science and Urban Economics, 35, 239-263. doi: 10.1016/j.regsciurbeco.2004.04.004

Urzúa, C. (2001). Las ciudades mexicanas no siguen la ley de Zipf. Estudios Demográficos y Urbanos, 16(3), 661-669.

Weziak, D. (2016). Quality of life in cities. Empirical evidence in comparative European perspective. Cities, 58, 87-96. doi: 10.1016/j.cities.2016.05.016

Zul, F., Hudalah, D., Rahayu, P., y Woltjer, J. (2014). Extended urbanization in small and medium-sized cities: The case of Cirebon. Indonesia. Habitat International, 42, 1-10. doi: 10.1016/j.habitatint.2013.10.003 